\title{
Quantitative analysis of complex nanocomposites based on straight skeletonization
}

\author{
Pablo G. Tahoces ${ }^{\mathrm{a}}$, Paula V. Messina ${ }^{\mathrm{b}}$, Juan M. Ruso ${ }^{\mathrm{c}, *}$ \\ ${ }^{a}$ Department of Electronic and Computation, University of Santiago de Compostela, Spain \\ ${ }^{\mathrm{b}}$ Department of Chemistry, Universidad Nacional del Sur, INQUISUR-CONICET, B8000CPB Bahía Blanca, Argentina \\ ${ }^{\mathrm{c}}$ Soft Matter and Molecular Biophysics Group, Department of Applied Physics, University of Santiago de Compostela, 15782, Spain
}

\section{G R A P H I C A L A B S T R A C T}

Example of the quntitative analysis of hydroxyapatite crystals.

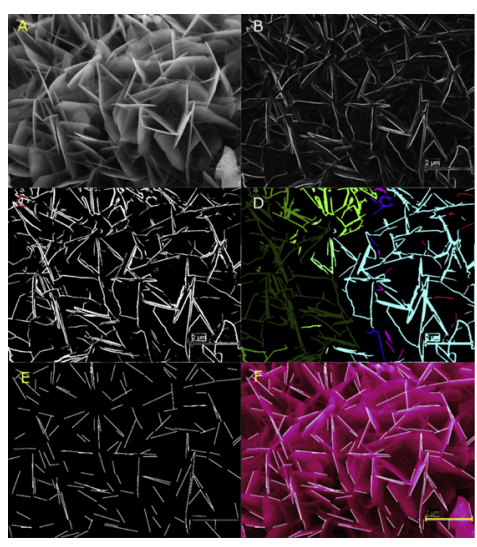

\section{A R T I C L E I N F O}

\section{Keywords:}

Hydroxyapatite

Bioceramics

Digital image analysis

Skeletonization

Nanoparticles

\begin{abstract}
A B S T R A C T
Bones are complex nanocomposites composed mainly by hydroxyapatite nanocrystals. Different factors characterize its morphology: composition, length, orientation, roughness. To increase our understanding of the tissue morphology at this fundamental lever of organization, a new method based on the straight skelonization of the images obtained by electronic microscopy is proposed. The method detects and measures the length and angularity of any straight edge of over the image. The technique resolved several test patterns independent of size and angle of rotation. Several samples obtained from different substrates were analyzed with the method. The results were consistent with those values obtained from conventional methods. Although still limited as a laboratory application, shape analysis has the potential to provide insight into the mechanisms of crystal growing and may provide a basis for specifications or guidelines for the manufacturing of biomaterial for bone tissue engineering. Our proposed automated computational method for the analysis and quantification digital images of bone tissue at microscale provide a rapid and accurate of the mechanical properties of the tissue.
\end{abstract}

\footnotetext{
* Corresponding author.

E-mail address: juanm.ruso@usc.es (J.M. Ruso).
} 


\section{Introduction}

The bones are complex nanocomposites scaffolds composed mainly by hydroxyapatite (HAP) nanocrystals arranged as parallel nanocrystals with the c-axis aligned with the long axis in a collagen-rich matrix. These nanocrystals have plate shapes with thicknesses of 2 to $7 \mathrm{~nm}$, lengths of 15 to $200 \mathrm{~nm}$, and widths from 10 to $80 \mathrm{~nm}$. The extraordinary properties of bone arise from its complex hierarchically structured geometry [1]. These structural features cover multiple length scales from molecular to almost macroscopic dimensions. The origin of many intrinsic mechanical properties lies in the smaller length scales: submicron to nanometers [2]. On the other hand, the bone morphology has to allow physiological functions such as osteoblasts migration, proliferation and differentiation. For this purpose, it has developed an extraordinary 3D architecture of interconnected pores. However, the origin of these self-assembled arrangements are not completely understood and the crystal structures of hydroxyapatite (HAP) and the nonmineralized collagen fibrils alone do not provide a complete explanation [3].

Disorders such as osteoporosis increase the probability of fractures. Assuming that mechanical integrity is the gold standard of bones, it is therefore important to take them into account and try to determine the relationships between mechanical properties and HAP morphology. It is believed that mechanical properties are strongly related with HAP composition, sintering routes, compaction, size and orientation but it is not well understood how changes occurring at this scale influence the mechanical properties at higher length scales [4]. The diagnosis of disorders related to bone loss involves determining bone density. For this purpose, the most commonly techniques used are bone densitometry scanning (dual-energy X-ray absorptiometry) and quantitative ultrasound (QUS) [5]. Despite the wide use of these powerful techniques they have several restrictions arising from the difficulties of covering the meso- and nano- dimensions or the huge variety of properties (sizes, shapes, physicochemical) involved in the systems. Besides, manual analysis involves several problems including: less reliability and feasibility, higher risks of negligence and mistakes, uncompleted coverage or time consuming. Consequently, alternative computational approaches may help to rationalize the assessment of these materials. Computer analysis guarantee: less time for test, repeatability, objectivity and reduced cost of assay.

Both techniques above mentioned involve the analysis of images. Digital image analysis has become increasingly widespread over the past decades and is used not only to collect data and represent it in the form of an image but also to enhance the information and avoid human subjectivity. Traditionally, the use of images has been restricted to the scope of the extraction of qualitative information, as would be the observation of the presence/absence of certain signs or the comparison between the shapes of certain structures. This way of using the images

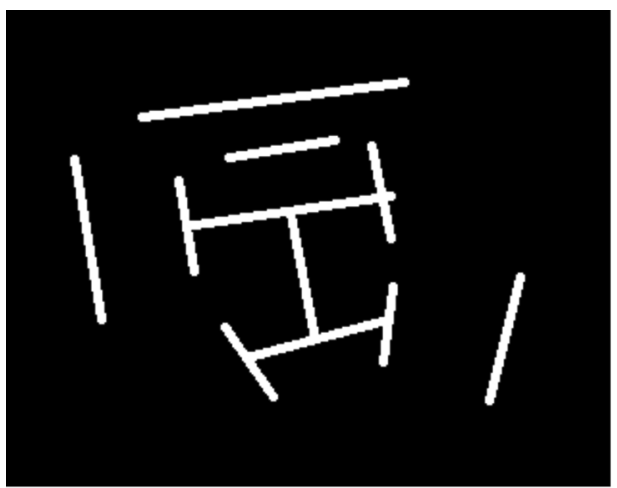

a) has the advantage of the immediacy, but the inconvenience of the subjectivity of the information extracted. The arrival of the digital format has opened the possibility of using the images as a quantitative tool, for the analysis of experimental results.

Complex image analysis is not a trivial task. In most cases it is necessary to transform such complex structures into a map of unique shapes, which can be modeled by mathematical analytic expressions. The skeletonization of the image is an important step in transforming complex images into a map composed of thin lines that follow the path of the structures that make up the original image. Our intention here is to develop a computer software to analyze and process images from HAP crystal with the aim of linking automatically (and discover) morphological parameters with mechanical properties of bone. Alongside, it could be possible to discover new relationships between the nano-arrangements and the macroscopic properties.

For this purpose, we have analyzed images obtained from different experiments. Within each type of experiment there is considerable flexibility in terms of choice of treatments, experimental conditions and time. We will focus on quantitative rather than qualitative data and subsequent statistical analysis. Finally, we think that this procedure not only will be useful in bone regeneration, but also in other health and materials areas where hierarchical structures will be present: tribology [6] or wetting [7].

\section{Methods}

\subsection{Concepts concerning the algorithm}

In many occasions, as the result of an experiment, the most relevant information obtained, is related with the distribution and orientation of certain regular shapes along an image. Lines, circles or ellipsoids in general, constitute in many cases a source of relevant information that is necessary to extract in order to establish conclusions about the result of an experiment. However, the process of extraction of such information can became tedious, non-reproducible or even none directly applicable.

The main objective of the proposed algorithm is to automate the process of extraction of information from images and more specifically, the extraction of information related with the presence, distribution and orientation of straight lines throughout the whole image. Examples of such kind of situations can be observed in Fig. 1.a, where a binary synthetic image composed by several lines is depicted. The algorithm has detected every line and has assigned to each detected line the equation of a straight line (Fig. 1.b). Thereby, each line belonging to the image it is fully characterized, thus their number, their length and their orientation can be now easily calculated.

However, usually lines are not explicitly present. It can be intuited, as part of the border of the 3D structures shown in the image (Fig. 2A).

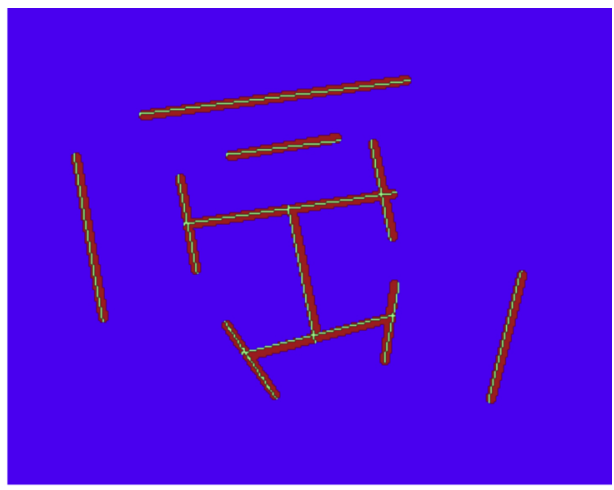

b)

Fig. 1. Example of binary images. 


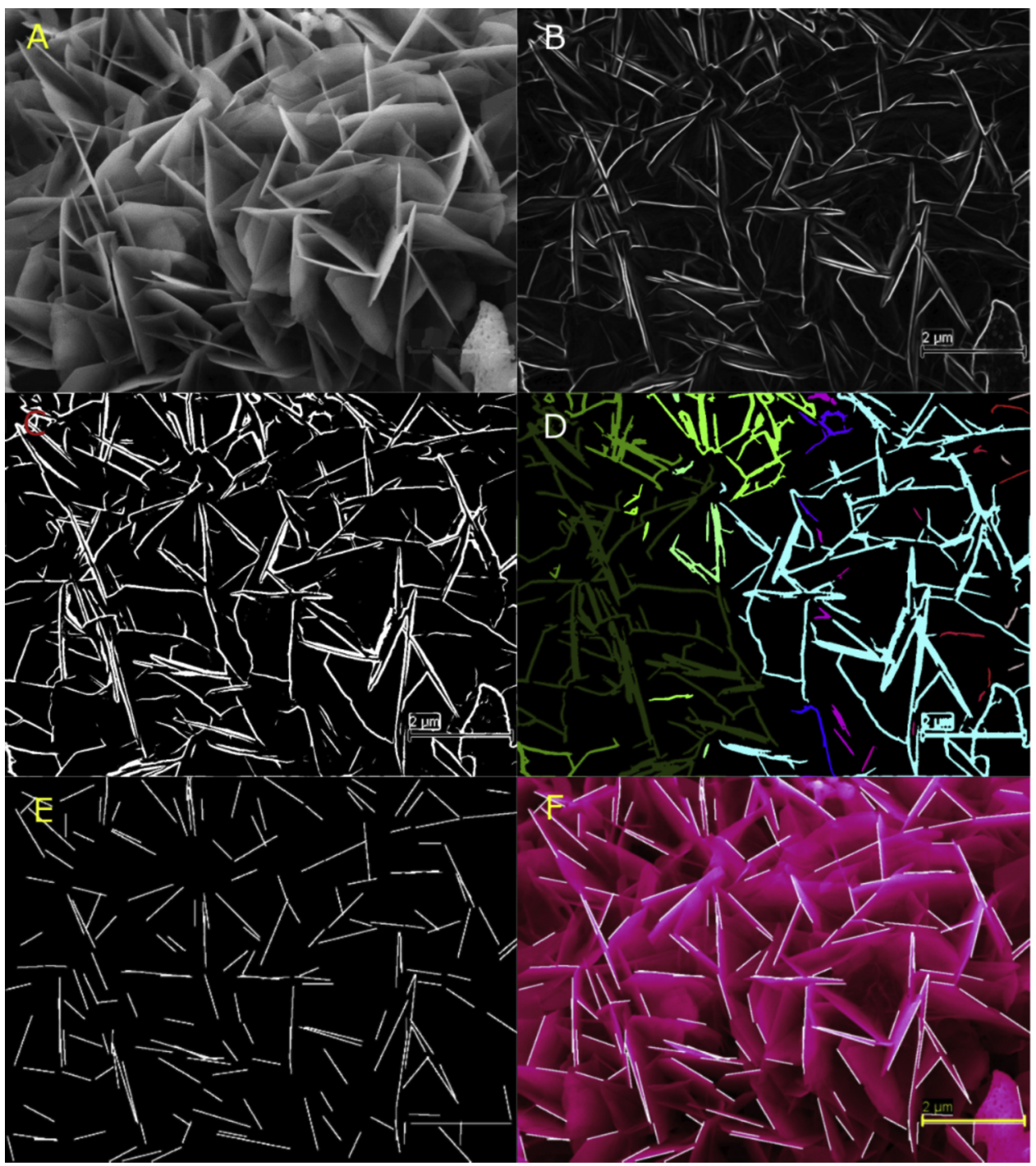

Fig. 2. A) Example of hydroxyapatite crystals. B) Impressing of Fig. $2 \mathrm{~A}$ after having applied a gradient filter. C) Impressing of Fig. 2B after thresholding. D) Image tagged. Each color represents an individual structure, which is not connected to the rest. E) Lines automatically extracted by the computer. F) Same lines superimposed on the original image.

Moreover, the background is not uniform, making it difficult to analyze the image by a human observer. The algorithm then, has first to transform the original images to synthetic images where the most relevant borders are represented as thin lines and then, over such synthetic image, perform an exhaustive search of such straight lines, to calculate its number, length and orientation.

The algorithm can be described by means of five main steps: i) Noise reduction, ii) structure enhancement, iii) thresholding, iv) artifacts removal and v) detection of straight lines detection.

i) Noise reduction - is an optional step. However, for complex images like Fig. 2 is advisable. In general, for these kinds of images, the noise plays an important role when filters for structures enhancement are applied. In such situations, if noise is not previously removed, certain artifacts can appear within the image, derived from this noise. If removal of noise is too aggressive, part of the structures that we want to detect could be removed. We have employed for this task an anisotropic filter. The main characteristic of this filter is that it works iteratively. It removes noise when perform in each iteration, but it preserves the shape of the structures.

ii) Structure Enhancement - previous to the detection of the structures inserted on the image, it is necessary to enhance such structures from the background. Different filters have been proposed in the literature for this task [8]. In our case we have employed a gradient filter that has the capability of enhancing the high contrast regions of the image that are related with the border of the structures (Fig. 2B).

iii) Thresholding - a threshold is then applied to transform the multitoned image to a binary map with just two values: one for border structures and zero for background (Fig. 2C). The main reason for this step is to simplify the detection of structures which is main task of the next step of the algorithm.

iv) Artifact removal - after applying the enhancement filter (step ii), some artifacts arise in the form of small disconnected structures. To remove such artifacts, a labeling process is performed. Thereby, each pixel in the image is assigned to a region where all the pixels are connected i.e. at least one of their eight neighbors should not belong to the background. Regions with a reduced number of pixels are considered artifacts and are removed in this step. Every region now has a label assigned (Fig. 2D) and will be individually process in the next step.

v) Straight Lines Detection - for each point in every labeled region, thirty-two straight lines equi-spaced, within an arc of $180^{\circ}$, are traced. Thereby, hundreds or even thousands of straight lines are 
Table 1

Average lengths achieved from the images obtained from the substrates indicated in the table.

\begin{tabular}{|c|c|c|c|c|}
\hline Time & $\begin{array}{l}\text { Avg Length }(\mu \mathrm{m}) \text { Manually Traced } \\
\text { Manually Measured } \\
\text { (MT / MM) }\end{array}$ & $\begin{array}{l}\text { Avg Length }(\mu \mathrm{m}) \text { Manually } \\
\text { Traced } \\
\text { Computer Measured } \\
\text { (MT / CM) }\end{array}$ & $\begin{array}{l}\text { Avg Length }(\mu \mathrm{m}) \text { Computer Traced } \\
\text { Computer Measured } \\
(\mathrm{CT} / \mathrm{CM})\end{array}$ & $\begin{array}{l}\text { Avg Length }(\mu \mathrm{m}) \text { Computer Traced } \\
\text { Computer Measured } \\
\text { Corrected by Model } \\
\text { (CT / CMC) }\end{array}$ \\
\hline \multicolumn{5}{|c|}{ Spongy nanostructured $\mathrm{SiO}_{2} /$ substrate template: NaDC-DDAB mixed vesicles [8] } \\
\hline 5 days & $1.09 \pm 0.25$ & $1.28 \pm 0.26$ & $0.90 \pm 0,23$ & $1.30 \pm 0.38$ \\
\hline 10 days & $0.94 \pm 0.25$ & $0.84 \pm 0.37$ & $0.60 \pm 0.25$ & $0.81 \pm 0.41$ \\
\hline 30 days & $1.20 \pm 0.36$ & $1.10 \pm 0.44$ & $0.71 \pm 0.29$ & $0.97 \pm 0.45$ \\
\hline \multicolumn{5}{|c|}{ HA nanorods / substrate template: CTAB-PPG rod-like mixed micelle [9] } \\
\hline 10 days & $0.15 \pm 0.03$ & $0.17 \pm 0.03$ & $0.16 \pm 0.04$ & $0.08 \pm 0.06$ \\
\hline \multicolumn{5}{|c|}{ HA nanorods / substrate template: CTAB-PEG rod-like mixed micelle [9] } \\
\hline 10 days & $0.12 \pm 0.02$ & $0.14 \pm 0.01$ & $0.15 \pm 0.03$ & $0.06 \pm 0.04$ \\
\hline \multicolumn{5}{|c|}{ HA nanorods / substrate template: CTAB/PEG-PPG-PEG rod-like mixed micelle [9] } \\
\hline 10 days & $0.20 \pm 0.04$ & $0.26 \pm 0.06$ & $0.24 \pm 0.04$ & $0.21 \pm 0.07$ \\
\hline \multicolumn{5}{|c|}{$\mathrm{TiO}_{2}$ nanoparticles / substrate template: water/CTAB/ButOH/n-heptane [10] } \\
\hline 10 days & $0.19 \pm 0.05$ & $0.24 \pm 0.04$ & $0.23 \pm 0.05$ & $0.20 \pm 0.08$ \\
\hline \multicolumn{5}{|c|}{ Striped $\mathrm{Ce}-\mathrm{TiO}_{2} /$ substrate template: water/CTAB/ButOH/Ce(Val $)_{3} / \mathrm{n}$-heptane } \\
\hline 10 days & $0.15 \pm 0.03$ & $0.20 \pm 0.03$ & $0.21 \pm 0.04$ & $0.17 \pm 0.06$ \\
\hline 21 days & $0.23 \pm 0.04$ & $0.27 \pm 0.02$ & $0.29 \pm 0.05$ & $0.30 \pm 0.08$ \\
\hline 24 days & $0.29 \pm 0.05$ & $0.31 \pm 0.05$ & $0.29 \pm 0.04$ & $0.29 \pm 0.06$ \\
\hline \multicolumn{5}{|c|}{$\mathrm{CeO}_{2}-\mathrm{TiO}_{2}$ nanoparticles / substrate template: water/CTAB/ButOH/n-heptane $+\mathrm{CeO}_{2}$ nanoparticles [21] } \\
\hline 60 days $(0.86 \%)$ & $1.20 \pm 0.41$ & $1.28 \pm 0.39$ & $0.90 \pm 0.26$ & $1.31 \pm 0.43$ \\
\hline $\begin{array}{l}60 \text { days } \\
\qquad(2.13 \%)\end{array}$ & $0.22 \pm 0.06$ & $0.29 \pm 0.04$ & $0.29 \pm 0.05$ & $0.30 \pm 0.09$ \\
\hline \multicolumn{5}{|l|}{ Mg doped [15] } \\
\hline 10 days & $0.07 \pm 0.01$ & & $0.13 \pm 0.01$ & $0.03 \pm 0.02$ \\
\hline
\end{tabular}

traced for each structure generated in the second step of the algorithm. Then, every straight line is iteratively compared with the rest of the straight lines of the same region, by checking the paths between its corresponding extremes. If none of the points of each path belong to the background, we can conclude that both lines belong to the same structure. The straight line, with the shortest length will be then eliminated. The lines that overcome this process, are supposed to be, the best representation of each structure generated, and its length and its slope are the length and slope of such structure. An example of how the algorithm works over a real image is show in Fig. 2E and $\mathrm{F}$ where the result of applying the algorithm on the image of the example is depicted.

\subsection{Experimental section}

\subsubsection{Reagents}

Sodium deoxycholate (NaDC), didodecyldimethylammonium bromide (DDAB), hexadecyl-trimethyl ammonium bromide (CTAB), titanium (IV) isopropoxide (TTIP, Ti (IV)(OiPr) $4, \delta=0.960 \mathrm{~g} \mathrm{~cm}^{-3}, 97 \%$ ), poly (propylene glycol) 400 (PPG400, $\delta=1.004 \mathrm{~g} \mathrm{~cm}^{-3}$ at $25^{\circ} \mathrm{C}$ ), polyvinyl alcohol (PVA, $119 \mathrm{~g} \mathrm{~cm}^{-3}$ ), poly (propylene glycol) (PPG, $\delta=1.004 \mathrm{~g} \mathrm{~cm}^{-3}$ at $25^{\circ} \mathrm{C}$ ), sodium phosphate $\left(\mathrm{Na}_{3} \mathrm{PO}_{4}, 96 \%\right)$, calcium chloride $\left(\mathrm{CaCl}_{2}, 99 \%\right)$, sodium nitrite $\left(\mathrm{NaNO}_{2}, 97 \%\right)$, sodium chloride $(\mathrm{NaCl}, 99,5 \%)$, sodium bicarbonate $\left(\mathrm{NaHCO}_{3}, 99,5 \%\right)$, potassium chloride (KCl, 99,5\%), di potassium phosphate tri-hydrate $\left(\mathrm{K}_{2} \mathrm{HPO}_{4} \cdot 3 \mathrm{H}_{2} \mathrm{O}, 99 \%\right)$, di-basic sodium phosphate $\left(\mathrm{Na}_{2} \mathrm{HPO}_{4}, 99,95 \%\right)$, magnesium chloride hexa-hydrate $\left(\mathrm{MgCl} .6 \mathrm{H}_{2} \mathrm{O}, 98 \%\right)$, sodium sulfate $\left(\mathrm{Na}_{2} \mathrm{SO}_{4}, 99 \%\right)$, chloride acid sodium hydroxide $(\mathrm{NaOH}, 97 \%)$ were obtained from Sigma- Aldrich and used as purchased. Butyl alcohol (ButOH, $\delta=0.810 \mathrm{~g} \mathrm{~cm}^{-3}$, Merck), n-heptane $\left(\delta=0.684 \mathrm{~g} \mathrm{~cm}^{-3}\right.$, Merck), cerium valerate $\left(\mathrm{Ce}(\mathrm{Val})_{3}\right)$ and $(\mathrm{HCl}, 30.0-34.0 \%$ Cicarelli) were of analytical grade. For solutions preparation, only triplet-distilled water was used

\subsubsection{Substrates}

Spongy nanostructured $\mathrm{SiO}_{2}, \mathrm{HAP}$ and $\mathrm{Mg}$-HAP nano-rods, striped Ce- $-\mathrm{TiO}_{2}, \mathrm{TiO}_{2}$ and $\mathrm{CeO}_{2}-\mathrm{TiO}_{2}$ nano cuboids were synthesized accordingly to previous works [9-11] using bile salts (NaDC/DDAB) mixed vesicles, $\mathrm{CTAB} /$ polymer mixed micelles and water/CTAB/ButOH/n- heptane microemulsions systems and their modifications as templates [12].

For mineralization assay, the samples were kept in contact with simulated body fluid (SBF) following the standard procedure described by Kokubo et al. [13,14] which has a non-protein ionic composition analogous to that of human plasma, containing $\mathrm{Na}^{+}(142.0 \mathrm{mM}), \mathrm{K}^{+}$ (5.0 mM), $\mathrm{Mg}^{2+}$ (1.5 mM), $\mathrm{Ca}^{2+}(2.5 \mathrm{mM}), \mathrm{Cl}^{-}$(148.8 mM), $\mathrm{HCO}^{3-}$ (4.2 mM), $\mathrm{HPO}_{4}{ }^{-2}(1.0 \mathrm{mM})$ and $\mathrm{SO}_{4}{ }^{-2}(0.5 \mathrm{mM})$. The synthesized materials were soaked in $1.5 \mathrm{SBF}$ at $37^{\circ} \mathrm{C}$ for periods of $5,10,15,30$ days, the specimens were removed from fluid, rinsed with distilled water and dried. The temperature $\left(37^{\circ} \mathrm{C}\right)$ was maintained by placing the samples in a thermostated bath throughout the experiment.

For mechanical characterization of mineralized ceramics, samples were prepared by traditional pressureless sintering method [15] Initially, 1\%wt polyvinyl alcohol (PVA) solution was mixed thoroughly with powder samples. Following, the mixed powders through 100 mesh sieves were biaxially compressed to cylindrical shape of $16.12 \pm 0.30 \mathrm{~mm}$ diameter and $13.80 \pm 0.20 \mathrm{~mm}$ height under the pressure of $100 \mathrm{MPa}$ and sintered at $1150-1250^{\circ} \mathrm{C}$ for $2 \mathrm{~h}$ under ambient atmosphere, with a heating rate of $\sim 10^{\circ} \mathrm{C} / \mathrm{min}$. After furnace cooled, to minimize internal cracks, all sintered samples were polished to a $1 \mathrm{~mm}$ finish prior to testing.

The compressive strength of cylindrical samples along the longitudinal direction were measured with a dynamical mechanical analyzer, Instron Universal Testing Machine (Model 3369) using a $5000 \mathrm{~N}$ load cell at a strain rate of $0.01 \mathrm{~mm} \mathrm{~s}^{-1}$.

\section{Results}

As we have pointed out in the previous section, the morphological characterization of HA crystals has traditionally been performed manually by measuring one by one each of the leaves in the images. In this section, the power of the quantitative shape analysis of the skeletonization algorithm proposed is experimentally tested. There are two main properties quantifying the HA morphology which are of major significance and which can be measured experimentally: length and orientation. For this purpose, we have chosen different images obtained from our own experiments and from general bibliography. Tables 1 and 2 show the mean values obtained for the length and the orientation 
Table 2

Average angles achieved from the images obtained from the substrates indicated in the table.

\begin{tabular}{|c|c|c|c|c|}
\hline Time & $\begin{array}{l}\text { Avg Angle (degrees) } \\
\text { Manually Traced } \\
\text { Computer Measured }\end{array}$ & $\begin{array}{l}\text { Avg Angle (degrees) } \\
\text { Computer Traced } \\
\text { Computer Measured }\end{array}$ & Kurtosis / Skewness & $\begin{array}{l}\text { Compressive } \\
\text { Modulus (MPa) }\end{array}$ \\
\hline \multicolumn{5}{|c|}{ Spongy nanostructured $\mathrm{SiO}_{2}$ / substrate template: NaDC-DDAB mixed vesicles [8] } \\
\hline 5 days & $101.33 \pm 52.26$ & $92.64 \pm 50.53$ & $-1.14 /-0.23$ & 42.1 \\
\hline 10 days & $88.75 \pm 63.99$ & $94.61 \pm 57.63$ & $-1.23 /-0.17$ & 39.5 \\
\hline 30 days & $81.95 \pm 57.75$ & $78.49 \pm 53.31$ & $-0.88 / 0.41$ & 41.9 \\
\hline \multicolumn{5}{|c|}{ HA nanorods / substrate template: CTAB-PPG rod-like mixed micelle [9] } \\
\hline 10 days & $95.28 \pm 50.45$ & $92.53 \pm 41.98$ & $-0.72 / 0.01$ & 30.4 \\
\hline 10 days & $104.58 \pm 48.69$ & $86.70 \pm 56.74$ & $-1.25 / 0.01$ & 31.2 \\
\hline \multicolumn{5}{|c|}{ HA nanorods / substrate template: CTAB/PEG-PPG-PEG rod-like mixed micelle [9] } \\
\hline 10 days & $81.99 \pm 62.60$ & $79.58 \pm 61.01$ & $-1.53 / 0.32$ & 30.8 \\
\hline \multicolumn{5}{|c|}{$\mathrm{TiO}_{2}$ nanoparticles / substrate template: water/CTAB/ButOH/n-heptane [10] } \\
\hline 10 days & $103.15 \pm 53.44$ & $89.52 \pm 53.39$ & $-0.28 /-0.11$ & 61.6 \\
\hline \multicolumn{5}{|c|}{ Striped Ce- $\mathrm{TiO}_{2} /$ substrate template: water/CTAB/ButOH/Ce(Val $)_{3} /$ n-heptane } \\
\hline 10 days & $91.46 \pm 53.44$ & $84.91 \pm 53.39$ & $-1.43 /-0.27$ & 69.5 \\
\hline 21 days & $109.89 \pm 40.61$ & $92.45 \pm 55.53$ & $-1.37 /-0.20$ & 69.0 \\
\hline 24 days & $95.76 \pm 46.55$ & $95.67 \pm 51.50$ & $-1.12 /-0.05$ & 69.3 \\
\hline \multicolumn{5}{|c|}{$\mathrm{CeO}_{2}-\mathrm{TiO}_{2}$ nanoparticles / substrate template: water/CTAB/ButOH/n-heptane $+\mathrm{CeO}_{2}$ nanoparticles [21] } \\
\hline 60 days $(0.86 \%)$ & $95.48 \pm 47.72$ & $99.71 \pm 49.40$ & $-1.06 /-0.26$ & 68.5 \\
\hline \multicolumn{5}{|l|}{$(2.13 \%)$} \\
\hline \multicolumn{5}{|l|}{ Mg doped [15] } \\
\hline 10 days & $93.47 \pm 50.89$ & $93.44 \pm 60.58$ & $-1.43 /-0.18$ & 67.9 \\
\hline
\end{tabular}

(kurtosis and skewness) respectively of HA crystals over different substrates. For length, we have included the results achieved by four different methods: i) Manually Traced / Manually Measured (MT / MM). In this case the selection of the straight structures of the image was made by hand, by means of an electronic pencil over a touch screen. Each structure was then measured, also by hand, with an electronic caliper. ii) Manually Traced / Computer Measured (MT / CM). Straight structures were obtained by hand, but the computer has automatically detected and measure such structures. iii) Computer Traced / Computer Measured (CT / CM). In this case, the computer was responsible, for the selection of the straight lines representatives of the structures of the image. Moreover, the computation of the length of each structure was also performed by the computer, without any human interaction. iv) Computer Traced / Computer Measured Corrected by Model (CT / CMC). Like iii), but in this case, before computer measurement, a calibration procedure was performed. Thereby, a mathematical model based in experimental results was built to adjust the zero value (the lower end of the measurent range) and the span (the algebraic difference between the upper and lower range values). We have to point out that the calibration procedure does not modify the correlation among the results obtained. Therefore, the correlation between CT /CM is the same as the achieve after calibration procedure is performed (CT / $\mathrm{CMC}$ ). For orientation analysis, for every image the histogram of the values of the angle of each line was obtained. Then, kurtosis and the skewness of such histograms were calculated as descriptors of the distribution (narrow or wide) of such lines orientation. Even though the lines over the image were independently traced both by hand and by the computer software, computation of the angles were performed only by the computer. Thus, results for MT / CM and CT / CM were solely presented (see Table 1). To make sure that this procedure does not disturb our conclusions, we have previously checked, in a small set of lines, that manual measurement of each angle is not only tedious, but hardly differ of the value obtained from the computer.

Fig. 3.a shows the correlation between both MT / MM and MT / CM methods. A correlation coefficient of 0.986 was achieved in this case. The measures obtained by both procedures drop over a straight line of $45^{\circ}$ of slope (red line), demonstrating the good agreement between both methods. Fig. 3b shows the correlation between both MT / MM (fully manual) and CT / CM (fully computer) methods. A correlation coefficient of 0.946 was achieved in this case. Thereby, a lineal relationship between both procedures is demonstrated. However, results obtained are different (but proportional) and a calibration procedure must be stablishing, to adjust zero and span (Column CT / CMC of Table 1). Worst results, after calibration, correspond with low quality images (see Table 1). CTAB-PEG rod-like mixed micelle and $\mathrm{Mg}$ doped (Fig. 4) are some clear examples. By the contrary, for most of the cases, the results achieved are encourageous (See Fig. 5).

In terms of angles (Table 2), the high dispersion of the results obtained, demonstrate the random spatial orientation of the structures extracted, for both manual and computer methods. This conclusion can be also established by the visual analysis of the images involved in the study.

\section{Discussion}

As shown in Table 1, both methods exhibit similar values and trends, it is noticeable the proximity between both set of data, the average difference between both methods is below $0.1 \mu \mathrm{m}$. Only samples with low quality images show significant discrepancy. Overall, this agreement suggests that the ideas involved in our method are helpful relating to the morphological characterization of nanostructured systems. We also believe that these results would be better if we had more quality images to analyze; this could be performed in future studies.

Here, the obligatory inquiry is the analysis of HA crystal length variations as a function of time and samples. Although the mechanism of hydroxyapatite coating formation in a biological environment on bioactive materials is not completely elucidated some of its characteristics are widely accepted such as: the evolution of the amorphous region and the crystallized region related to the crystallization of amorphous phase is a diffusion-controlled process, driven mainly by the calcium and phosphorous ion diffusion rates [12,16,17]; or the thickness of the HA coating increases with time and reaches a saturated point after around ten days of soaking $[18,19]$. For these reasons most of the studies focus on the evolution of the coating during the first ten days, as can be observed in Table 1. Only longer times (20 or 60 days) were used for samples doped with $\mathrm{Ce}$, because these materials were intended as oxygen storage systems (Ce has a great ability to scavenge free radicals [20]) for industrial applications, instead of medical applications. All these elements in addition to the results listed in Table 1 and the bibliography [21] allow us to conclude that the definitive length of the HA crystals is reached after two or three days, a time less than needed for saturation, as we have already pointed out. Once the final size is 


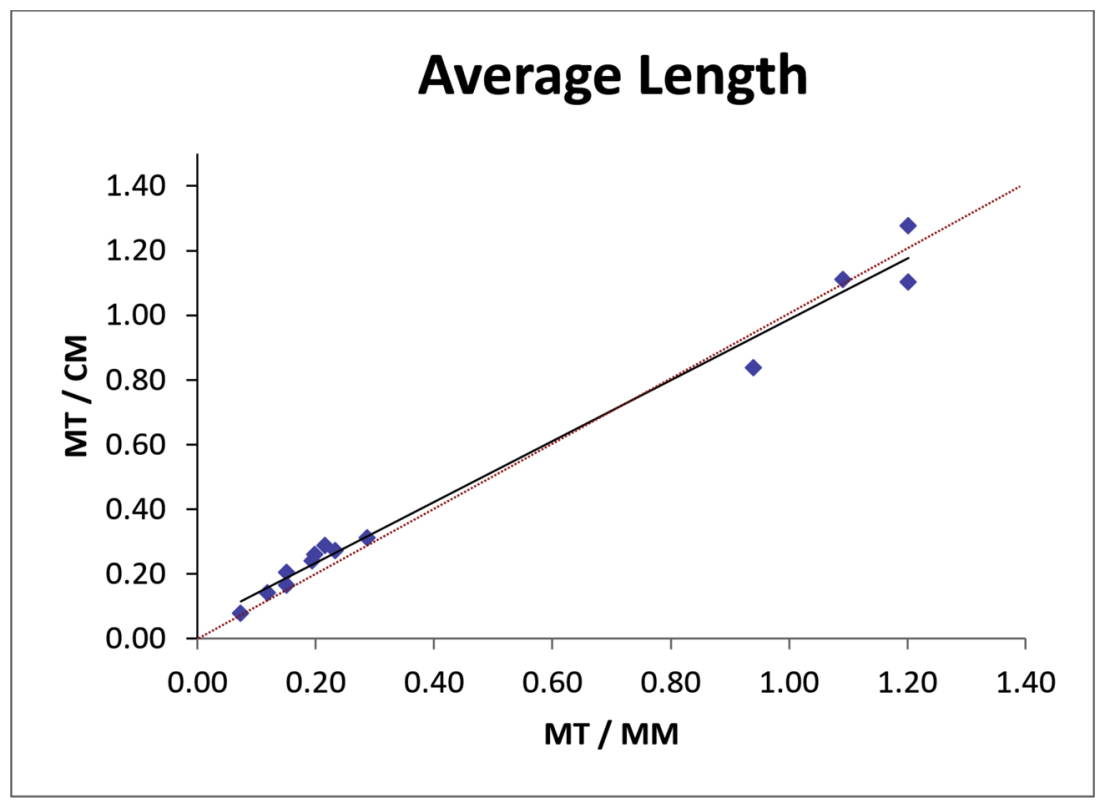

Fig. 3. a. Plot of the Average Length obtained by the methods MT / MM vs MT / CM. Black line corresponds with the straight line obtained after lineal adjustment. Red line is a straight line of $45^{\circ}$ slope, included for comparison. b. Plot of the Average Length obtained by the methods MT / MM vs CT / CM. Black line corresponds with the straight line obtained after lineal adjustment. Red line is a straight line of $45^{\circ}$ slope, included for comparison (For interpretation of the references to colour in this figure legend, the reader is referred to the web version of this article).
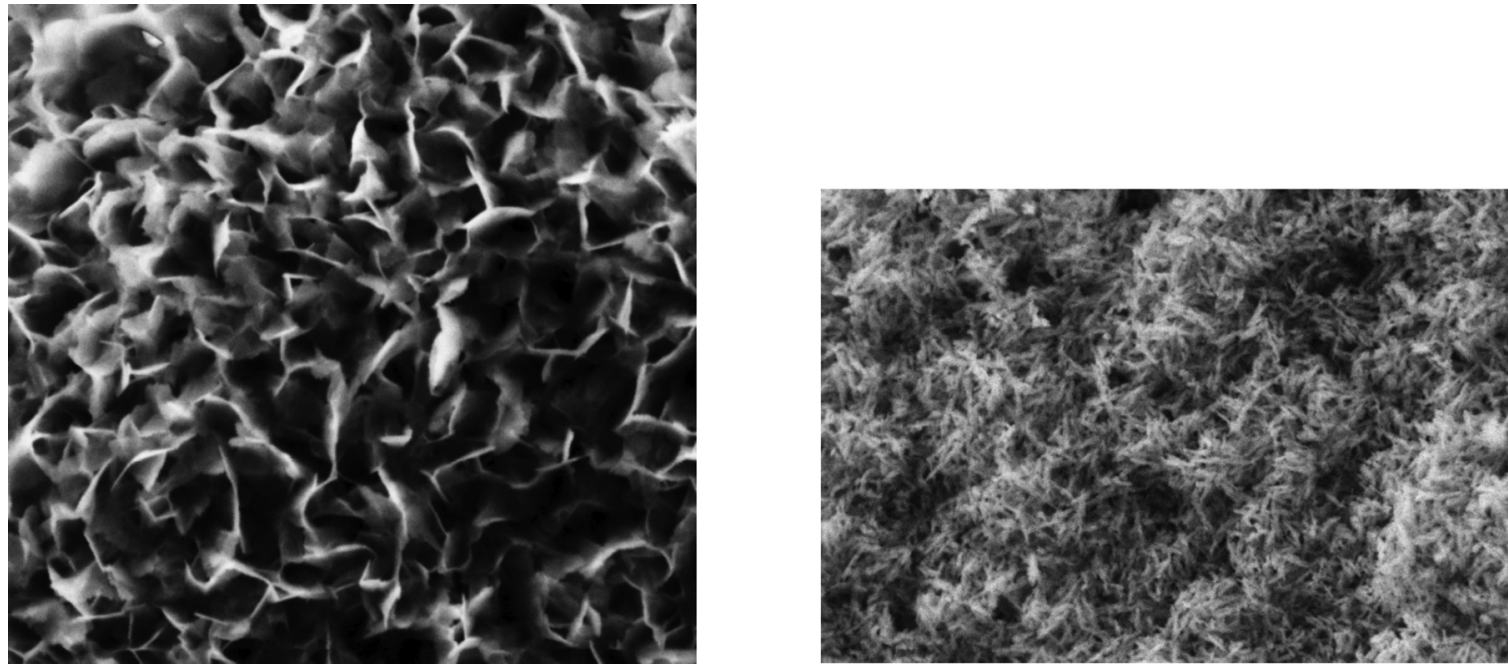

Fig. 4. Low quality image examples. HA nanorods / substrate template CTAB-PPG rod-like mixed micelle (left) and Mg doped (right).

reached, the variations are minimal and fall within the uncertainty of the measures.

Another interesting point is the ability of the substrate to influence the maximum length of the HA crystals. According to the results obtained it seems obvious that the substrate templated with bile salts
(NaDC-DDAB) are what generate the largest crystals. Although Cedoped $\mathrm{TiO}_{2}$ nanoparticles also give very large values, two factors can be the cause of this: on the one hand the large time involved ( 60 days) and on the other the influence that the atoms of cerium have on the final morphology of the samples, deforming the HA crystal lattice which
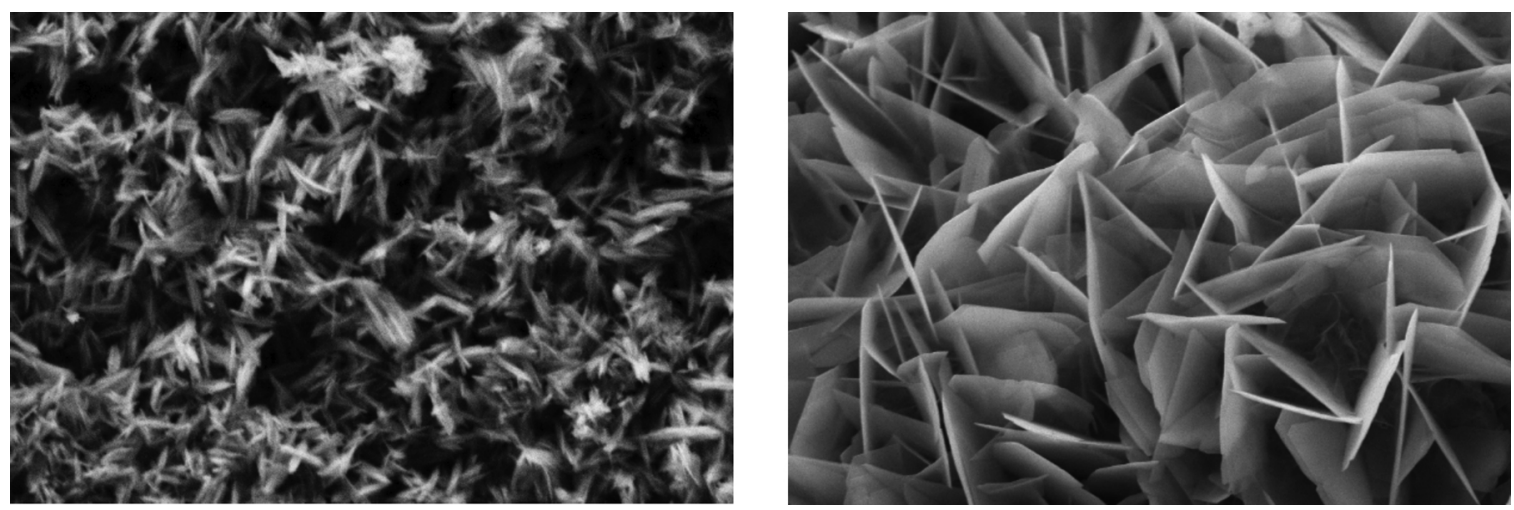

Fig. 5. left Striped Ce-TiO 2 (left) and Spongy nanostructured $\mathrm{SiO}_{2}$ (right). 

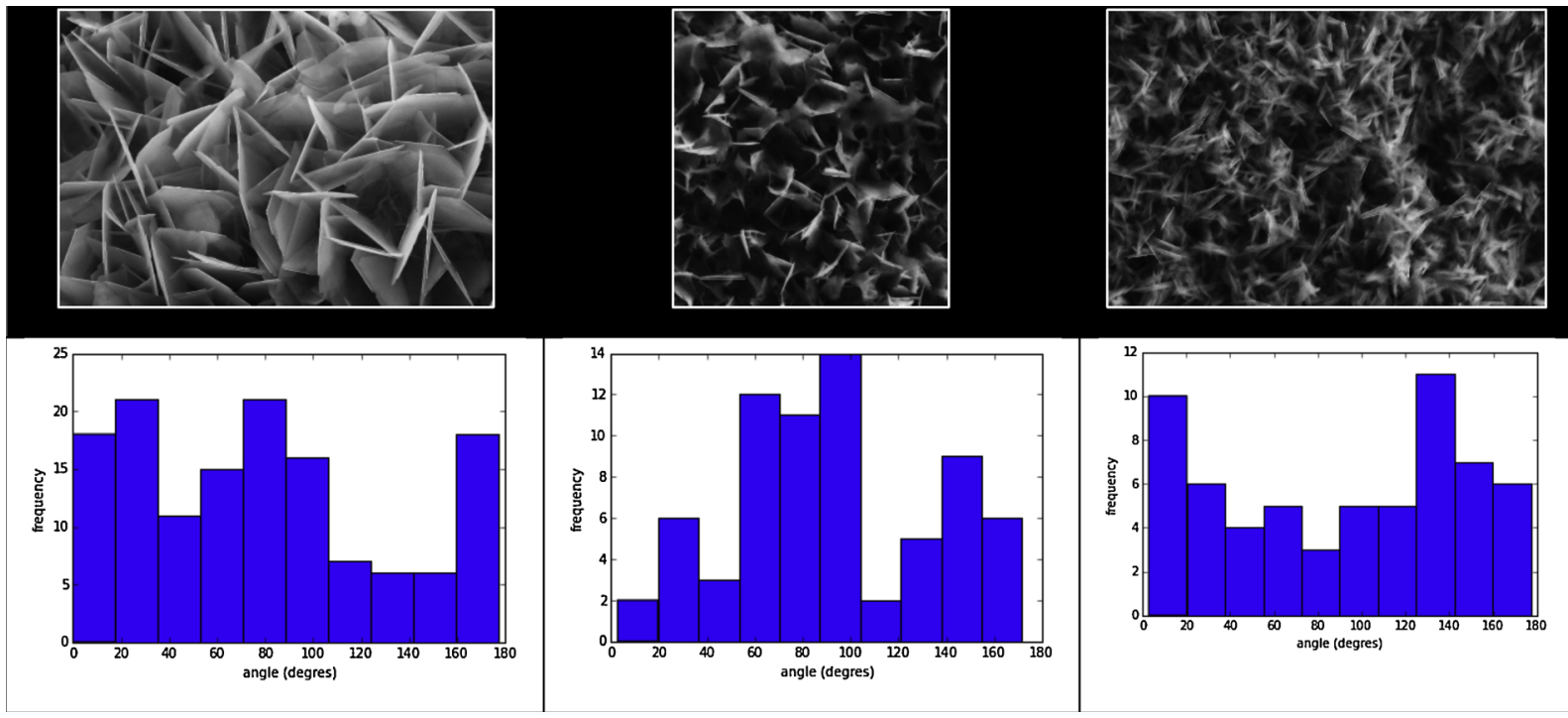

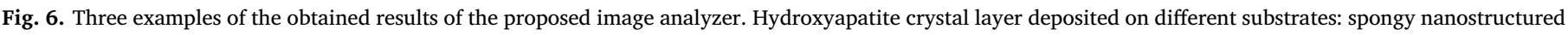

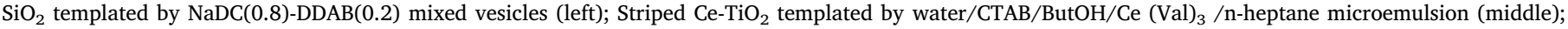
HAP nano-rods template by CTAB/PPG mixed micelles (right). Top line: Crystal picture. Bottom line: slope histograms.

results in the formation of fullerene-like structures. Previous publications have shown that titanium has a good catalytic ability to favor the nucleation and growth of hydroxyapatite. However, it is important to note that the effectiveness of the crystal growth does not depend only on the composition but also on the structural arrangements. In the case of titanium, the efficiency of growth depends on the mineral form present: apatite is the most efficient followed by rutile and brookite. Consequently, the length of the crystals depends on the concentration of these phases, which depend enormously on the route of synthesis [22]. Going back to the bile salts, this templates under specific synthesis conditions generated an open bioactive mesoporous architecture with a tunnel-like lattice very similar to those presenting the trabecular bone [23].

In order to get insight into the crystal orientation in two dimensions, our software also allows to obtain the distribution of the main orientations (expressed in angles) of the HA crystals. The value of the average angle and histograms obtained can be consulted in Table 2 and Fig. 6, respectively. Table 2 shows the average values that were obtained automatically. In this case it can be detected a greater homogeneity than that obtained for the lengths. The mean average values for all the samples under study were found to be $91^{\circ}$. However, as displayed in the histograms plotted in Fig. 6, due to a relatively wide range of the distributions obtained, one cannot simply establish the validity of the mean value. In the liquid crystal state, nematic and smectic phases the molecules tend to point in the same direction, whereas in the liquid phase they are randomly arranged. Steric effect such as dipole-dipole interactions, exclude volume effect, dispersion forces or hydrogen bonding promote this alignment [24,25]. Consequently, we attribute the disorder in the orientation of the crystals to the strong contribution of ionic and covalent bonds that shield any steric interaction.

The morphological characteristics of the surface of the samples can be known through two statistical parameters: skewness and kurtosis. Positive values of skewness correspond to a surface with wide valleys and narrow peaks. If the values are negative the profiler of the surface will be exactly the otherwise. The kurtosis is related to the sharpness of the surface, if it is greater than three the peaks and the depressions will be narrow and sharp; if it is less than three peaks and valleys will be wide and blunt. Our results reveal some patterns. Kurtosis is always negative; this means wide grooves and blunt peaks. Skewness values are mostly negative, and the positives ones are very close to zero. The mean value of kurtosis was higher than the mean skewness value. It is clear that differences in values in both quantities cause a different crystal nucleation and growth. Surface texturing that results in a larger and more negative kurtosis and skewness is characterized by a wider spacing between the peaks and seems to be the preferred sites for the nucleation and growth of HA crystals [26].

As we have already commented the mechanical properties of the bone depend on many factors being the most determinant the volume fraction of the elements of the mineral phase. However, recently Jimenez-Palomar et al. [27] shew the important relevance that the mineral platelet orientation has in strength and elastic modulus of the bone. Although this study focused on a higher hierarchical scale, it highlights the importance that spatial organization at the micro and nano level has on macroscopic properties such as stiffness and elasticity. The distribution of the angles we obtained from our samples did not reveal specific orientations, so in our case we consider that the most relevant variable could be the length of the crystals. For this purpose, we carry out uniaxial compression measurements within controlled environmental conditions. The stress versus strain graphics showed that all materials have similar behavior: linear response at low deformations and plastic deformation at higher ones. A similar pattern has been found in bone tissue samples $[28,29]$. This deviation of linearity has its origin in the crystallographic properties of the hydroxyapatite, that not being an atomic crystal experience more limits to deformation along certain crystallographic directions [30]. The obtained compressive moduli for the samples are listed in Table 2 . It can be observed that time hardly has influence on the stiffness of the samples. On the other side, there is an increased stiffness when nanoparticles (TiO2) are incorporated, revealed by the expansion of the compressive modulus. Previous studies have shown that the effect of nanoparticles on the mechanical properties is very heterogeneous [31,32]. The addition of carbon nanotubes [33] or graphene oxide [34] had almost no impact on the stiffness of the material. However, the system composed by poly(3hydroxibutyrate) and HA nanoparticles improves the mechanical properties with HA concentration [35]. Thus, the mechanical properties will depend on the degree of the component interlocking. In the former example the nanoparticles act simply as fillers that did not increase the compressive modulus of the sample. The later correspond to a system with strong interfacial hydrogen bonds between HA and the polymer causing a good stress.

Finally, focusing in the first three systems, $\mathrm{SiO}_{2}$ and $\mathrm{HAP}$ substrates templated by bile salts and polymers PPG and PEG, it is evident that the 
length of the crystals has a clear effect on the mechanical properties: longer crystals produce more rigid materials.

\section{Conclusions}

In this study, we have developed an automated computational method for the analysis and quantification of the main morphological characteristics of hydroxyapatite nanostructures from two-dimensional digital images. These characteristics includes meaningful measurements of length, orientations, kurtosis and skewness. Among the most outstanding novelties of the proposed algorithm, one can highlight the detection and elimination of artifacts, and the ability to discern between overlapping straights or belonging to the same structure, in ways that automatically Identifies and selects the longest or most representative of the area. The software was checked with varying structures and images of different quality. In all cases, the results of our software were always better than those obtained manually or semiautomatic. Finally, we have related a macroscopic property as the rigidity with the parameters obtained from the analysis of the images. We are aware that the number of images used is relatively limited and the lack of a general conclusion linking the microscopic characteristics analyzed with macroscopic properties, such as hardness, of the materials. However, as the image analysis becomes more popular, our method will become a very useful tool to extract information in an objective and automatic way, increasing the existing databases, thus allowing obtaining of conclusions of a general nature. Finally, to comment that this software, in addition to its use in bone regeneration materials, could be used in other areas of great interest such as electrospinning, textile industry or 3D printing.

Lastly we want to remark that, for reproducibility purposes, we have included the software and some examples we used as experimental setup on the website https://persoal.citius.usc.es/pablo.tahoces/ demoSL/, as part of our contribution.

\section{Acknowledgements}

The authors acknowledge Fundación Ramón Areces, Xunta de Galicia (ED431B 2017/21), MINECO project reference TIN2016-76373P (AEI/FEDER, UE), Universidad Nacional del Sur (PGI 24/Q064), CONICET PIP-11220130100100CO.

\section{References}

[1] N. Reznikov, R. Shahar, S. Weiner, Bone hierarchical structure in three dimensions, Acta Biomater. 10 (2014) 3815-3826.

[2] R.O. Ritchie, The conflicts between strength and toughness, Nat. Mater. 10 (2011) 817-822.

[3] J. Tao, K.C. Battle, H. Pan, E.A. Salter, Y.-C. Chien, A. Wierzbicki, J.J. De Yoreo, Energetic basis for the molecular-scale organization of bone, Proc. Natl. Acad. Sci. 112 (2015) 326-331.

[4] M.A. Hammond, J.M. Wallace, Exercise prevents [beta]-aminopropionitrile-induced morphological changes to type I collagen in murine bone, Bonekey Rep. 4 (2015).

[5] E.M. Lochmüller, D. Bürklein, V. Kuhn, C. Glaser, R. Müller, C.C. Glüer, F. Eckstein, Mechanical strength of the thoracolumbar spine in the elderly: prediction from in situ dual-energy X-ray absorptiometry, quantitative computed tomography (QCT), upper and lower limb peripheral QCT, and quantitative ultrasound, Bone 31 (2002) $77-84$.

[6] M. Sedlaček, B. Podgornik, J. Vižintin, Correlation between standard roughness parameters skewness and kurtosis and tribological behaviour of contact surfaces, Tribol. Int. 48 (2012) 102-112.

[7] A. Sauret, F. Boulogne, B. Soh, E. Dressaire, H.A. Stone, Wetting morphologies on randomly oriented fibers, Eur. Phys. J. E 38 (2015) 1-9.

[8] R.C. González, R.E. Woods, Digital Image Processing, third edition ed., Pearson Prentice Hall, 2018.

[9] M. Fernández-Leyes, V. Verdinelli, N. Hassan, J.M. Ruso, O. Pieroni, P.C. Schulz, P. Messina, Biomimetic formation of crystalline bone-like apatite layers on spongy materials templated by bile salts aggregates, J. Mater. Sci. 47 (2012) 2837-2844.

[10] N.L. D’Elía, A.N. Gravina, J.M. Ruso, J.A. Laiuppa, G.E. Santillán, P.V. Messina, Manipulating the bioactivity of hydroxyapatite nano-rods structured networks: effects on mineral coating morphology and growth kinetic, Biochim. Biophys. Acta (BBA) - Gen. Subj. 1830 (2013) 5014-5026.

[11] A.N. Gravina, J.M. Ruso, J.A. Laiuppa, G.E. Santillan, J.L. Marco-Brown, N.L. D'Elia, P.V. Messina, Striped, bioactive Ce-TiO2 materials with peroxynitritescavenging activity, J. Mater. Chem. B 2 (2014) 834-845.

[12] N.C. Andrés, N.L. D’Elía, J.M. Ruso, A.E. Campelo, V.L. Massheimer, P.V. Messina, Manipulation of $\mathrm{Mg} 2+-\mathrm{Ca} 2+$ switch on the development of bone mimetic hydroxyapatite, ACS Appl. Mater. Interfaces 9 (2017) 15698-15710.

[13] T. Kokubo, H. Kushitani, S. Sakka, T. Kitsugi, T. Yamamuro, Solutions able to reproduce in vivo surface-structure changes in bioactive glass-ceramic A-W3, J. Biomed. Mater. Res. 24 (1990) 721-734.

[14] ISO, Implants for Surgery - In Vitro Evaluation for Apatite-Forming Ability of Implant Materials vol. 23317, (2014), p. 13.

[15] J. Song, Y. Liu, Y. Zhang, L. Jiao, Mechanical properties of hydroxyapatite ceramics sintered from powders with different morphologies, Mater. Sci. Eng. A 528 (2011) 5421-5427.

[16] A.L. Oliveira, S.A. Costa, R.A. Sousa, R.L. Reis, Nucleation and growth of biomimetic apatite layers on 3D plotted biodegradable polymeric scaffolds: effect of static and dynamic coating conditions, Acta Biomater. 5 (2009) 1626-1638.

[17] Y.-P. Lu, Y.-M. Chen, S.-T. Li, J.-H. Wang, Surface nanocrystallization of hydroxyapatite coating, Acta Biomater. 4 (2008) 1865-1872.

[18] P. Soares, C.A.H. Laurindo, R.D. Torres, N.K. Kuromoto, O. Peitl, E.D. Zanotto, Effect of a bioactive glass-ceramic on the apatite nucleation on titanium surface modified by micro-arc oxidation, Surf. Coat. Technol. 206 (2012) 4601-4605.

[19] X. Rao, J. Li, X. Feng, C. Chu, Bone-like apatite growth on controllable macroporous titanium scaffolds coated with microporous titania, J. Mech. Behav. Biomed. Mater. 77 (2018) 225-233.

[20] J.M. Dowding, S. Seal, W.T. Self, Cerium oxide nanoparticles accelerate the decay of peroxynitrite (ONOO-), Drug Deliv. Transl. Res. 3 (2013) 375-379.

[21] V. Dhand, K.Y. Rhee, S.-J. Park, The facile and low temperature synthesis of nanophase hydroxyapatite crystals using wet chemistry, Mater. Sci. Eng. C 36 (2014) $152-159$

[22] T. Kokubo, H.-M. Kim, M. Kawashita, Novel bioactive materials with different mechanical properties, Biomaterials 24 (2003) 2161-2175.

[23] R. Huiskes, R. Ruimerman, G.H. van Lenthe, J.D. Janssen, Effects of mechanical forces on maintenance and adaptation of form in trabecular bone, Nature 405 (2000) 704.

[24] M. Reufer, H. Dietsch, U. Gasser, A. Hirt, A. Menzel, P. Schurtenberger, Morphology and orientational behavior of silica-coated spindle-type hematite particles in a magnetic field probed by small-angle X-ray scattering, J. Phys. Chem. B 114 (2010) 4763-4769.

[25] T.P. Bigioni, X.-M. Lin, T.T. Nguyen, E.I. Corwin, T.A. Witten, H.M. Jaeger, Kinetically driven self assembly of highly ordered nanoparticle monolayers, Nat. Mater. 5 (2006) 265.

[26] P.S. Vanzillotta, M.S. Sader, I.N. Bastos, Gd.A. Soares, Improvement of in vitro titanium bioactivity by three different surface treatments, Dent. Mater. 22 (2006) 275-282.

[27] I. Jimenez-Palomar, A. Shipov, R. Shahar, A.H. Barber, Structural orientation dependent sub-lamellar bone mechanics, J. Mech. Behav. Biomed. Mater. 52 (2015) 63-71.

[28] J.-Y. Rho, L. Kuhn-Spearing, P. Zioupos, Mechanical properties and the hierarchical structure of bone, Med. Eng. Phys. 20 (1998) 92-102.

[29] D.T. Reilly, A.H. Burstein, The elastic and ultimate properties of compact bone tissue, J. Biomech. 8 (1975) 393-405.

[30] A. Zamiri, S. De, Mechanical properties of hydroxyapatite single crystals from nanoindentation data, J. Mech. Behav. Biomed. Mater. 4 (2011) 146-152.

[31] J. Corona-Gomez, X. Chen, Q. Yang, Effect of nanoparticle incorporation and surface coating on mechanical properties of bone scaffolds: a brief review, J. Funct. Biomater. 7 (2016) 18.

[32] J.R. Xavier, T. Thakur, P. Desai, M.K. Jaiswal, N. Sears, E. Cosgriff-Hernandez, R. Kaunas, A.K. Gaharwar, Bioactive nanoengineered hydrogels for bone tissue engineering: a growth-factor-Free approach, ACS Nano 9 (2015) 3109-3118.

[33] S.R. Shin, S.M. Jung, M. Zalabany, K. Kim, P. Zorlutuna, Sb. Kim, M. Nikkhah, M. Khabiry, M. Azize, J. Kong, K.-t. Wan, T. Palacios, M.R. Dokmeci, H. Bae, $\mathrm{X}$. Tang, A. Khademhosseini, Carbon-nanotube-Embedded hydrogel sheets for engineering cardiac constructs and bioactuators, ACS Nano 7 (2013) 2369-2380.

[34] S.R. Shin, B. Aghaei-Ghareh-Bolagh, T.T. Dang, S.N. Topkaya, X. Gao, S.Y. Yang, S.M. Jung, J.H. Oh, M.R. Dokmeci, X. Tang, A. Khademhosseini, Cell-laden microengineered and mechanically tunable hybrid hydrogels of gelatin and graphene oxide, Adv. Mater. 25 (2013) 6385-6391.

[35] J. Ramier, T. Bouderlique, O. Stoilova, N. Manolova, I. Rashkov, V. Langlois, E. Renard, P. Albanese, D. Grande, Biocomposite scaffolds based on electrospun poly(3-hydroxybutyrate) nanofibers and electrosprayed hydroxyapatite nanoparticles for bone tissue engineering applications, Mater. Sci. Eng. C 38 (2014) 161-169. 